Pacific

Journal of

Mathematics

\title{
SUMS OF SQUARES \\ IN ALGEBRAIC FUNCTION FIELDS \\ OVER A COMPLETE DISCRETELY VALUED FIELD
}

KaRim Johannes Becher, DaVid GRIMm AND JAN VAN GeEL 


\title{
SUMS OF SQUARES \\ IN ALGEBRAIC FUNCTION FIELDS OVER A COMPLETE DISCRETELY VALUED FIELD
}

\author{
Karim Johannes Becher, David Grimm and Jan VAn GeEl
}

\begin{abstract}
A recently found local-global principle for quadratic forms over function fields of curves over a complete discretely valued field is applied to the study of quadratic forms, sums of squares, and related field invariants.
\end{abstract}

\section{Introduction}

Let $K$ be a field of characteristic different from 2 and $F / K$ an algebraic function field (i.e., a finitely generated extension of transcendence degree one). The study of quadratic forms over $F$ is generally difficult, even in such cases where the quadratic form theory over all finite extensions of $K$ is well understood. It can be considered complete in the cases where $K$ is algebraically closed, real closed, or finite, but it is wide open for example when $K$ is a number field.

A breakthrough was obtained recently in the situation where the base field $K$ is a nondyadic local field. Parimala and Suresh [2010] proved that in this case any quadratic form of dimension greater than eight over $F$ is isotropic. Harbater, Hartmann, and Krashen [Harbater et al. 2009] obtained the same result as a consequence of a new local-global principle for isotropy of quadratic forms over $F$. The local conditions are in geometric terms, relative to an arithmetic model for $F$. A less geometric version of the local-global principle, in terms of the discrete rank one valuations of $F$, was obtained by Colliot-Thélène, Parimala, and Suresh [ColliotThélène et al. 2012]; see Theorem 6.1 below. Both versions of the local-global principle hold more generally when $K$ is complete with respect to a nondyadic discrete valuation.

In this article we apply the local-global principle to study sums of squares in $F$ and to obtain further results on quadratic forms over $F$. This is of particular interest in the case where $K$ is the field of Laurent series $k((t))$ over a (formally) real field $k$.

We outline the structure of this article and the main results. Section 2 provides some necessary basic results on valuations. In Section 3 we discuss discrete

MSC2010: primary 11E04, 11E10, 11E25, 12J10, 14H05; secondary 12D15, 12F20.

Keywords: isotropy, local-global principle, real field, sums of squares, $u$-invariant, Pythagoras number, valuation, algebraic function fields. 
valuations on an algebraic function field over a complete discretely valued field and characterize their residue fields. In Section 4 we move on to the study of sums of squares in fields and corresponding field invariants, in the context of valuations. In Section 5 we do an analogous discussion of the $u$-invariant in the context of valuations. According to [Elman and Lam 1973], the $u$-invariant of a field is the supremum on the dimension of anisotropic torsion forms over that field. In Section 6 we finally apply the local-global principle to obtain new results for algebraic function fields and in particular the rational function field. Let us describe some of these results.

In Theorem 6.4 we show that the upper bound on the dimension of anisotropic torsion forms over algebraic function fields over $K$ is the double of the corresponding upper bound for algebraic function fields over $k$, the residue field of the discrete valuation on $K$. We thus obtain an upper bound on the $u$-invariant of an algebraic function field over $K$. We obtain in Theorem 6.6 a refinement for the case of the rational function field, saying that the $u$-invariant of $K(X)$ is equal to the supremum of the $u$-invariant of all $\ell(X)$ where $\ell / k$ is a finite algebraic extension. In Corollary 6.9 we prove that the Pythagoras number of the rational function field $K(X)$ is equal to the supremum of the Pythagoras numbers of $\ell(X)$ for all finite field extensions $\ell / k$. We conjecture in Conjecture 4.16 that this is equal to the Pythagoras number of $k(X)$. This is motivated by the observation - made previously in [Scheiderer 2001] — that both Pythagoras numbers are bounded by the same power of two. In the case where $k$ is real closed we show in Theorem 6.12 that any sum of squares in $F$ can be expressed as a sum of three squares and further prove the finiteness of $\sum F^{2} / D_{F}(2)$, the quotient of the group of nonzero sums of squares modulo the subgroup of sums of two squares in $F$.

Our methods are based on valuation theory and quadratic form theory, for which [Engler and Prestel 2005] and [Lam 2005] are our standard references. We also use some algebraic geometry, namely desingularization of arithmetic surfaces and the properties of blowing-ups in regular points. Our reference on this topic is [Liu 2002].

This article grew out of results obtained in the Ph.D. thesis of D. Grimm under the supervision of K. J. Becher at Universität Konstanz.

\section{Valuations}

For a ring $R$ we denote by $R^{\times}$its group of invertible elements.

Let $K$ be a field. Given a valuation on $K$, we denote by $O_{v}$ the valuation ring of $v$, by $\mathfrak{m}_{v}$ its maximal ideal, by $\kappa_{v}$ the residue field, by $K^{v}$ the completion of $K$ with respect to $v$, and we further call $v$ dyadic if $\kappa_{v}$ has characteristic 2, nondyadic otherwise. Given a local ring $R$ contained in $K$, we say that a valuation $v$ of $K$ dominates $R$ if $\mathfrak{m}_{v} \cap R$ is the maximal ideal of $R$. Given a field extension $L / K$, we say that a valuation $v$ of $L$ is unramified over $K$ if $v\left(L^{\times}\right)=v\left(K^{\times}\right)$. 
A valuation with value group $\mathbb{Z}$ is called a $\mathbb{Z}$-valuation. Any discrete valuation of rank one can be identified (via a unique isomorphism of the value groups) with a $\mathbb{Z}$-valuation. A commutative ring is the valuation ring of a $\mathbb{Z}$-valuation if and only if it is a regular local ring of dimension one (see [Matsumura 1986, Theorem 11.2]); such rings are called discrete valuation rings.

Lemma 2.1. Let $w_{1}$ and $w_{2}$ be two valuations on $K$ such that $\mathfrak{m}_{w_{1}} \subseteq \mathcal{O}_{w_{2}}$. Then $\mathrm{O}_{w_{1}} \subseteq \mathrm{O}_{w_{2}}$ or $\mathrm{O}_{w_{2}} \subseteq \mathrm{O}_{w_{1}}$.

Proof. If $\mathfrak{m}_{w_{1}} \subseteq \mathfrak{m}_{w_{2}}$, then $\mathfrak{O}_{w_{1}} \supseteq \mathfrak{O}_{w_{2}}$, otherwise for any choice of $t \in \mathfrak{m}_{w_{1}} \backslash \mathfrak{m}_{w_{2}}$ we have $t^{-1} \in \mathcal{O}_{w_{2}}$ and $\mathcal{O}_{w_{1}}=t^{-1}\left(t \mathcal{O}_{w_{1}}\right) \subseteq t^{-1} \mathfrak{m}_{w_{1}} \subseteq \mathcal{O}_{w_{2}}$.

The property for a valuation to be henselian is characterized by a list of equivalent conditions, including the statement of Hensel's Lemma, hence satisfied in particular by complete $\mathbb{Z}$-valuations; see [Engler and Prestel 2005, Section 4.1].

Proposition 2.2. Let $v$ be a henselian $\mathbb{Z}$-valuation on $K$. Then $v$ is the unique $\mathbb{Z}$-valuation on $K$.

Proof. By [Engler and Prestel 2005, Corollary 2.3.2] for distinct $\mathbb{Z}$-valuations $w_{1}$ and $w_{2}$ on $K$ one has $\mathrm{O}_{w_{1}} \nsubseteq \mathrm{O}_{w_{2}}$ and $\mathrm{O}_{w_{2}} \nsubseteq \mathrm{O}_{w_{1}}$. Consider now a $\mathbb{Z}$-valuation $w$ on $K$. Since $v$ is henselian we have $1+\mathfrak{m}_{v} \subseteq K^{\times^{n}}$ for all $n \in \mathbb{N}$ prime to the characteristic of $\kappa_{v}$. As $w\left(K^{\times}\right)=\mathbb{Z}$, this implies that $1+\mathfrak{m}_{v} \subseteq \mathbb{O}_{w}^{\times}$and thus $\mathfrak{m}_{v} \subseteq \mathfrak{O}_{w}$. Now Lemma 2.1 yields that $\mathfrak{O}_{w}=\mathfrak{O}_{v}$.

Let $X$ always denote a variable over a given ring or field.

Proposition 2.3. Let $R$ be a local domain with maximal ideal $\mathfrak{m}$ and residue field $k$. Let $p \in R[X]$ be monic and such that $\bar{p} \in k[X]$, the reduction of $p$ modulo $\mathfrak{m}$, is irreducible. Then $R[X] /(p)$ is a local domain with maximal ideal $(\mathfrak{m}[X]+(p)) /(p)$ and residue field $k[X] /(\bar{p})$. The ring $R[X] /(p)$ has the same dimension as $R$. Moreover, if $R$ is regular, then $R[X] /(p)$ is regular.

Proof. Note that $\mathfrak{m}[X]+(p)$ is a maximal ideal of $R[X]$. Consider a maximal ideal $M$ of $R[X]$ containing $p$ and set $\mathfrak{p}=M \cap R$. Since $R[X] /(p)$ is an integral extension of $R$, it follows using [Matsumura 1986, Theorems 9.3 and 9.4] that both rings have the same dimension. Moreover, the field $R[X] / M$ is an integral extension of $R / \mathfrak{p}$, whereby $R / \mathfrak{p}$ is a field. It follows that $\mathfrak{p}=\mathfrak{m}$ and thus $M=\mathfrak{m}[X]+(p)$. This shows that $\mathfrak{m}[X]+(p)$ is the unique maximal ideal of $R[X]$ containing $p$. Hence, $R[X] /(p)$ is a local domain with maximal ideal $(\mathfrak{m}[X]+(p)) /(p)$ and residue field $k[X] /(\bar{p})$. Any set of generators of $\mathfrak{m}$ in $R$ yields a set of generators of $(\mathfrak{m}[X]+(p)) /(p)$ in $R[X] /(p)$. In particular, if $R$ is regular, so is $R[X] /(p)$.

Corollary 2.4. Let $T$ be a discrete valuation ring of $K$ with residue field $k$. Let $p \in T[X]$ be monic with $\bar{p} \in k[X]$ irreducible. Then $T[X] /(p)$ is a discrete valuation ring with field of fractions $K[X] /(p)$ and residue field $k$-isomorphic to $k[X] /(\bar{p})$. 
Proof. Since a discrete valuation ring is the same as a regular local ring of dimension one, the statement follows from Proposition 2.3.

We want to mention the following partial generalization of Corollary 2.4.

Proposition 2.5. Let $T$ be a valuation ring of $K$ with residue field $k$ and let $\ell / k$ be a finite field extension. There exists a finite field extension $L / K$ with $[L: K]=[\ell: k]$ and $a$ valuation $v$ on $L$ dominating $T$ and unramified over $K$ whose residue field is $k$-isomorphic to $\ell$.

Proof. It suffices to consider the case where $\ell=k[x]$ for some $x \in \ell$. Let $\mathfrak{m}$ denote the maximal ideal of $T$. Let $p \in T[X]$ be a monic polynomial whose residue $\bar{p}$ in $k[X]$ is the minimal polynomial of $x$ over $k$. Then $p$ is irreducible in $K[X]$, so $L=K[X] /(p)$ is a field. We obtain from Proposition 2.3 that $R=T[X] /(p)$ is a local domain with maximal ideal $M=(\mathfrak{m}[X]+(p)) /(p)$ and residue field $k[X] /(\bar{p})$. Let $v$ be a valuation on $L$ dominating $T$. Then $T \subseteq R \subseteq \mathrm{O}_{v}$, and as $M$ is generated by $\mathfrak{m}$, it follows that $v$ dominates $R$. Hence, $k[X] /(\bar{p})$ embeds naturally into $\kappa_{v}$. In particular $\left[\kappa_{v}: k\right] \geq \operatorname{deg}(\bar{p})=\operatorname{deg}(p)=[L: K]$. Using the Fundamental Inequality [Engler and Prestel 2005, Theorem 3.3.4] we conclude that $v$ is unramified over $K$ and $\left[\kappa_{v}: k\right]=\operatorname{deg}(\bar{p})=[L: K]$, whereby $\kappa_{v}$ is $k$-isomorphic to $k[X] /(\bar{p})$ and therefore to $\ell$.

\section{Valuations on algebraic function fields}

In this section we want to relate algebraic function fields over a valued field to algebraic function fields over the corresponding residue field. In particular we show in Proposition 3.4 that an algebraic function field over the residue field of a valuation on $K$ can be realized as the residue field of an unramified extension to some algebraic function field over $K$, and we refine this statement in Theorem 3.5 for rational function fields.

In the sequel let $T$ denote a valuation ring, $K$ its field of fractions, and $k$ the residue field of $T$. (That is, we have $T=\mathrm{O}_{v}$ for a valuation $v$ on $K$ and $k=\kappa_{v}$.) We consider the residue fields of valuations dominating $T$. (The reader may observe that we avoid to speak of extensions of valuations, as this can lead to confusion about the corresponding value groups.) For a field extension $F / K$ and a valuation $v$ on $F$ dominating $T$, the field $k$ is naturally embedded in the residue field $\kappa_{v}$. We often identify residue fields of valuations dominating $T$ up to $k$-isomorphism, in order to simplify the language.

A finitely generated field extension $F / K$ of transcendence degree one is called an algebraic function field. We say that $F / K$ is an algebrorational function field if $F=L(x)$ for a finite extension $L / K$ with $L \subseteq F$ and some element $x \in F$ that 
is transcendental over $L$; if this holds already with $L=K$, then $F / K$ is called $a$ rational function field.

Proposition 3.1. Let $F / K$ be an algebraic function field and $v$ a valuation on $F$ dominating $T$. The extension $\kappa_{v} / k$ is either algebraic or an algebraic function field.

Proof. This is a special case of the Dimension Inequality [Engler and Prestel 2005, Theorem 3.4.3].

The following is a refinement of Proposition 3.1 for rational function fields.

Theorem 3.2 (Ohm and Nagata). Let $F / K$ be a rational function field and $v$ be a valuation on $F$ dominating $T$. Then $\kappa_{v} / k$ is either algebraic or algebrorational.

Proof. This is shown in [Ohm 1983, Theorem], as a generalization of [Nagata 1967, Theorem 1].

We recall a construction to extend a valuation to a rational function field; in [Engler and Prestel 2005, Section 2.2] this is called the Gauss extension.

Proposition 3.3. Let $F / K$ be a rational function field. Let $x \in F$ be such that $F=K(x)$. Let $T^{\prime}$ be the localization of $T[x]$ with respect to the prime ideal $\mathfrak{m}[x]$ where $\mathfrak{m}$ is the maximal ideal of $T$. Then $T^{\prime}$ is a valuation ring with field of fractions $F$. The residue field of $T^{\prime}$ is $k(\bar{x})$ where $\bar{x}$ is the class of $x$ modulo $\mathfrak{m}[x]$, which is transcendental over $k$. The corresponding valuation $v$ on $F$ with $\mathrm{O}_{v}=T^{\prime}$, uniquely determined up to equivalence, is unramified over $K$.

Proof. This follows from [Engler and Prestel 2005, Corollary 2.2.2].

Proposition 3.4. Let $E / k$ be an algebraic function field. There exists an algebraic function field $F / K$ and a valuation $v$ on $F$ dominating $T$ and unramified over $K$ whose residue field is $E$.

Proof. Let $F^{\prime} / K$ be a rational function field. Let $x \in F^{\prime}$ be such that $F^{\prime}=K(x)$ and let $T^{\prime}$ denote the valuation ring described in Proposition 3.3. We identify $\bar{x}$ with some element of $E$ transcendental over $k$. Then $E / k(\bar{x})$ is a finite extension. By Proposition 2.5 there exists a finite field extension $F / F^{\prime}$ with $\left[F: F^{\prime}\right]=[E: k(\bar{x})]$ and a valuation $v$ on $F$ dominating $T^{\prime}$ and unramified over $F^{\prime}$ with residue field $E$. Using Proposition 3.3 it follows that $v$ is also unramified over $K$.

Theorem 3.5. Assume that $T \neq K$ and let $F / K$ be a rational function field. Let $\ell / k$ be a finite separable field extension. There exists a valuation $v$ on $F$ dominating $T$ and unramified over $K$ for which $\kappa_{v} / k$ is an algebrorational function field with field of constants $\ell$.

Proof. Let $y \in F$ and $\alpha \in \ell$ be such that $F=K(y)$ and $\ell=k(\alpha)$. Let $q \in T[Y]$ be monic and such that the residue $\bar{q}$ in $k[Y]$ is the minimal polynomial of $\alpha$. Let $\mathfrak{m}$ be the maximal ideal of $T$. We choose $m \in \mathfrak{m} \backslash\{0\}$ and set $x=m^{-1} q(y) \in F$. Note 
that $x$ is transcendental over $K$, and thus $F / K(x)$ is a finite extension. Let $T^{\prime}$ be the localization of $T[x]$ with respect to $\mathfrak{m}[x]$, the ideal consisting of the polynomials in $x$ with coefficients in $\mathfrak{m}$. Let $\mathfrak{m}^{\prime}$ be the maximal ideal of $T^{\prime}$. By Proposition 3.3 $T^{\prime}$ is a valuation ring with field of fractions $K(x)$ and residue field $k(\bar{x})$, and $\bar{x}$ is transcendental over $k$. Note that $\bar{q}$ remains irreducible in $k(\bar{x})[Y]$.

Consider $p=q-q(y) \in T^{\prime}[Y]$. As $q(y)=m x$, taking residues modulo $\mathfrak{m}^{\prime}[Y]$ we have $\bar{p}=\bar{q}$ in $k(\bar{x})[Y]$. It follows by Proposition 2.3 that $R=T^{\prime}[Y] /(p)$ is a local ring with maximal ideal lying over $\mathfrak{m}^{\prime}$, with field of fractions $K(x)[Y] /(p)$, and residue field $k(\bar{x})[Y] /(\bar{p})$. Note that $K(x)[Y] /(p)$ is $K(x)$-isomorphic to $F$. Using Chevalley's theorem [Engler and Prestel 2005, Theorem 3.1.1], we obtain a valuation $v$ on $F$ that dominates $T^{\prime}$. Then $v$ also dominates $T$. As $p(y)=0$, we have that $y$ is integral over $T^{\prime}$. Since $\bar{p}=\bar{q}$ is irreducible in $k(\bar{x})[Y]$, we have that $\bar{p}(0) \neq 0$, whereby $p(0) \in T^{\prime \times}$. As $v$ dominates $T^{\prime}$ and $p(y)=0$, we obtain that $v(y)=0$. Hence, $\bar{x}, \bar{y} \in \kappa_{v}$, and $\bar{y}$ is algebraic over $k$, because $\bar{q}(\bar{y})=\bar{p}(\bar{y})=0$. As $\bar{q}$ is irreducible in $k(\bar{x})[Y]$ we obtain that

$$
\left[\kappa_{v}: k(\bar{x})\right] \geq[k(\bar{x})[\bar{y}]: k(\bar{x})]=\operatorname{deg}(\bar{p})=\operatorname{deg}(p)=[F: K(x)] .
$$

By the Fundamental Inequality [Engler and Prestel 2005, Theorem 3.3.4], it follows that $v$ is unramified over $K(x)$ and $\kappa_{v}=k(\bar{x})[\bar{y}]=k[\bar{y}](\bar{x})$. Using Proposition 3.3 we obtain that $v$ is unramified over $K$. Since $q(\bar{y})=0=\bar{q}(\alpha)$ and since we consider residue fields up to $k$-isomorphism, we can identify $\ell=k[\alpha]$ with $k[\bar{y}]$.

Remark 3.6. In Theorem 3.5, the hypothesis on the finite extension $\ell / k$ to be separable is not necessary. Given a finite extension $\ell / k$ we can obtain a regular model (see below for the definition) for $F / T$ whose special fiber contains a component isomorphic to $\mathbb{P}_{\ell}^{1}$ in the following way: We choose $\alpha \in \ell$ and $\ell^{\prime}=k(\alpha)$. Blowing up $\mathbb{P}_{T}^{1}$ in a point on the special fiber $\mathbb{P}_{k}^{1}$ with residue field $\ell^{\prime}$, we obtain a new regular model whose special fiber has a component given by the exceptional fiber of this blowing-up and thus isomorphic to $\mathbb{P}_{\ell^{\prime}}^{1}$. Iterating this process we eventually obtain a regular model for $F / T$ whose special fiber has a component isomorphic to $\mathbb{P}_{\ell}^{1}$, and its generic point corresponds to a $\mathbb{Z}$-valuation whose residue field is a rational function field over $\ell$.

Assume that the valuation ring $T$ is discrete and consider an algebraic function field $F / K$. By a regular model for $F / T$ we mean a 2-dimensional integral regular projective flat $T$-scheme $\mathscr{X}$ whose function field is $K$-isomorphic to $F$. Given a regular model $\mathscr{X}$ for $F / K$ we denote by $\mathscr{X}_{k}$ its special fiber; by [Liu 2002, Chapter 8, Lemma 3.3] $\mathscr{X}_{k}$ is a curve.

Given an integral scheme $\mathscr{X}$, a point $P \in \mathscr{X}$, and a valuation $v$ on the function field of $\mathscr{X}$, we say that $v$ is centered at $P$ if $v$ dominates $\mathcal{O}_{\mathscr{X}, P}$, the local ring at $P$. 
Proposition 3.7. Assume that $T$ is a discrete valuation ring. Let $F / K$ be an algebraic function field. Let $\mathscr{L}$ be a regular model for $F / T$. Let $v$ be a $\mathbb{Z}$-valuation on $F$ dominating $T$. Then $v$ is centered at a point $P$ of $\mathscr{X}$ lying in $\mathscr{X}_{k}$. Moreover, if the extension $\kappa_{v} / k$ is neither algebraic nor algebrorational, then $\mathrm{O}_{v}=\mathrm{O}_{\mathscr{X}, P}$ where $P$ is the generic point of an irreducible component of $\mathscr{X}_{k}$.

Proof. By [Liu 2002, Chapter 8, Definition 3.17] $v$ is centered at a point $P$ of the special fiber $\mathscr{X}_{k}$. Since $\mathscr{X}_{k}$ is a curve, $P$ is either a closed point or the generic point of an irreducible component $\mathscr{X}_{k}$. In either case $\mathscr{O}_{\mathscr{X}, P}$ is a regular local ring.

If $P$ is a closed point of $\mathscr{X}_{k}$, then by [Abhyankar 1956, Proposition 3] the extension $\kappa_{v} / k$ is either algebraic or algebrorational. Assume now that $P$ is a generic point of $\mathscr{X}_{k}$. Then $P$ has codimension one in $\mathscr{X}$, so $\mathscr{O}_{\mathscr{X}, P}$ is a regular local ring of dimension one and thus a discrete valuation ring. As $\mathcal{O}_{\mathscr{X}, P}$ is dominated by $\mathrm{O}_{v}$ and both are discrete valuation rings with the same field of fractions, it follows by [Engler and Prestel 2005, Corollary 2.3.2] that $\mathcal{O}_{v}=\mathcal{O}_{\mathscr{X}, P}$.

Proposition 3.8. Assume that $T$ is a complete discrete valuation ring. Let $F / K$ be an algebraic function field. Then there exists a regular model for $F / T$.

Proof. There exists a regular projective curve $C$ over $K$ whose function field is $K$-isomorphic to $F$. If the curve $C$ is smooth, then by [Liu 2002, Chapter 10, Proposition 1.8)] there exists a regular model for $F / T$. Note that this applies in particular when $\operatorname{char}(K)=0$. Without assuming that $C$ is smooth, we can follow the first steps in the proof of the proposition cited to obtain a 2-dimensional projective $T$-scheme $\mathscr{X}$ with function field $F$. Since the structure morphism $\mathscr{X} \rightarrow \operatorname{Spec}(T)$ is surjective, it is flat (see [Liu 2002, Chapter 8, Definition 3.1]). Then $T$ is an excellent ring (see [ibid., Corollary 2.40]), and $\mathscr{X}$, being locally of finite type over $T$, is excellent (see [ibid., Theorem 2.39]).

Let $\mathscr{X}^{\prime} \rightarrow \mathscr{X}$ be the normalization of $\mathscr{X}$. Since $\mathscr{X}$ is excellent and projective over $T$, the normalization $\mathscr{X}^{\prime} \rightarrow \mathscr{X}$ is a finite projective birational morphism (see [ibid., Theorem 8.2.39 and Lemma 3.47]). The singular locus of $\mathscr{X}^{\prime}$ is closed in $\mathscr{X}^{\prime}$ (see [ibid., Corollary 2.38]). We consider the blowing-up $\mathscr{X}^{\prime \prime} \rightarrow \mathscr{X}^{\prime}$ along the singular locus of $\mathscr{X}^{\prime}$; this is a birational projective morphism (see [ibid., Propositions 1.12 and 1.22]).

We may alternate normalization and blowing-up until we reach a scheme that is regular. At each step we obtain a flat projective 2-dimensional $T$-scheme whose function field is $F$. By Lipman's desingularization theorem (see [ibid., Theorem 3.44]), after finitely many steps we come to a situation where the $T$-scheme is regular.

Corollary 3.9. Assume that $T$ is a complete discrete valuation ring. Let $F / K$ be an algebraic function field. Then there exist only finitely many $\mathbb{Z}$-valuations $v$ on $F$ dominating $T$ for which the extension $\kappa_{v} / k$ is neither algebraic nor algebrorational. 
Proof. By Proposition 3.8 there exists a regular model for $F / T$. The statement follows by applying Proposition 3.7 to any such model.

The result Corollary 3.9 can be extended to the situation where $T$ is an arbitrary discrete valuation ring. Moreover, one may ask to characterize the $\mathbb{Z}$-valuations on an algebraic function field that dominate a given discrete valuation ring of the base field and for which the residue field extension is neither algebraic nor algebrorational. We intend to develop these topics in a forthcoming article.

\section{Sums of squares and valuations}

From now on let $K$ be a field of characteristic different from 2. We denote by $\sum K^{2}$ the subgroup of nonzero sums of squares in $K$ and, for $n \in \mathbb{N}$, by $D_{K}(n)$ the set of nonzero elements that can be written as sums of $n$ squares in $K$. One calls

$$
s(K)=\inf \left\{n \in \mathbb{N} \mid-1 \in D_{K}(n)\right\} \in \mathbb{N} \cup\{\infty\}
$$

the level of $K$. Recall that $K$ is real if $s(K)=\infty$ and nonreal otherwise, and in the latter case $s(K)$ is a power of two (see [Lam 2005, Chapter XI, Section 2]).

The Pythagoras number of $K$ is defined as

$$
p(K)=\inf \left\{n \in \mathbb{N} \mid D_{K}(n)=\sum K^{2}\right\} \in \mathbb{N} \cup\{\infty\} .
$$

We further define

$$
p^{\prime}(K)= \begin{cases}p(K) & \text { if } K \text { is real, } \\ s(K)+1 & \text { if } K \text { is nonreal. }\end{cases}
$$

This field invariant has no independent interest, but it allows us to avoid case distinctions in statements about valuations and Pythagoras numbers, by formulating them for $p^{\prime}(K)$ rather than for $p(K)$. As for nonreal field $K$ we always have $s(K) \leq p(K) \leq s(K)+1=p^{\prime}(K)$. Hence, $p^{\prime}(K)$ is always equal to $p(K)$ or to $p(K)+1$.

We now consider valuations in the context of sums of squares. We say that a valuation $v$ on $K$ is real or nonreal, respectively, if the residue field $\kappa_{v}$ has the corresponding property.

Lemma 4.1. Let $v$ be a valuation on $K$ and $n \in \mathbb{N}$. Then $s\left(\kappa_{v}\right) \geq n$ if and only if $v\left(a_{1}^{2}+\cdots+a_{n}^{2}\right)=2 \min \left\{v\left(a_{1}\right), \ldots, v\left(a_{n}\right)\right\}$ holds for all $a_{1}, \ldots, a_{n} \in K$.

Proof. Both conditions are easily seen to be equivalent to having that any sum of $n$ squares of elements in $\mathbb{O}_{v}^{\times}$lies in $\mathbb{O}_{v}^{\times}$.

Let $\Omega(K)$ denote the set of nondyadic $\mathbb{Z}$-valuations on $K$.

Proposition 4.2. Let $v \in \Omega(K)$. If $v$ is real, then $v\left(\sum K^{2}\right)=2 \mathbb{Z}$. If $v$ is nonreal, then for $s=s\left(\kappa_{v}\right)$ we have $v\left(D_{K}(s)\right)=2 \mathbb{Z}$ and $v\left(D_{K}(s+1)\right)=v\left(\sum K^{2}\right)=\mathbb{Z}$. 
Proof. If $v$ is real, then it follows from Lemma 4.1 that $v\left(\sum K^{2}\right)=2 \mathbb{Z}$. Assume now that $v$ is nonreal and let $s=s\left(\kappa_{v}\right)$. Then it follows from Lemma 4.1 that $v\left(D_{K}(s)\right)=2 \mathbb{Z}$ and that there exist $x_{0}, \ldots, x_{s} \in K$ such that $v\left(x_{0}^{2}+\cdots+x_{s}^{2}\right) \neq$ $2 \min \left\{v\left(x_{0}\right), \ldots, v\left(x_{s}\right)\right\}$. Dividing by one of the elements $x_{0}, \ldots, x_{s}$ with minimal value, we can assume that $\min \left\{v\left(x_{0}\right), \ldots, v\left(x_{s}\right)\right\}=0$. Hence $v\left(x_{0}^{2}+\cdots+x_{s}^{2}\right) \geq 1$. If $v\left(x_{0}^{2}+\cdots+x_{s}^{2}\right)>1$, then we choose $t \in K$ with $v(t)=1$, and we conclude that $v\left(\left(x_{0}+t\right)^{2}+x_{1}^{2} \cdots+x_{s}^{2}\right)=1$, as $v$ is nondyadic. We may therefore assume that $v\left(x_{0}^{2}+\cdots+x_{s}^{2}\right)=1$. Since $K^{\times 2} \cdot D_{K}(s+1)=D_{K}(s+1)$, we conclude that $v\left(D_{K}(s+1)\right)=\mathbb{Z}$, and thus in particular that $v\left(\sum K^{2}\right)=\mathbb{Z}$.

Proposition 4.3. Let $v \in \Omega(K)$. Then $p^{\prime}(K) \geq p(K) \geq p^{\prime}\left(\kappa_{v}\right)$. Moreover, if $v$ is henselian, then $p^{\prime}(K)=p(K)=p^{\prime}\left(\kappa_{v}\right)$.

Proof. Note that $p(K) \geq p\left(\kappa_{v}\right)$. If $v$ is real, then $\kappa_{v}$ and $K$ are real, and we obtain that $p^{\prime}(K)=p(K) \geq p\left(\kappa_{v}\right)=p^{\prime}\left(\kappa_{v}\right)$. If $v$ is nonreal, then for $s=s\left(\kappa_{v}\right)$ we conclude that $D_{K}(s) \subsetneq D_{K}(s+1)$ by Proposition 4.2, and therefore $p^{\prime}(K) \geq p(K) \geq s+1=$ $p^{\prime}\left(\kappa_{v}\right)$.

Assume finally that $v$ is henselian. Then $s(K)=s\left(\kappa_{v}\right)$, and further $p(K)=p\left(\kappa_{v}\right)$ in case $v$ is real. This yields that $p^{\prime}(K)=p^{\prime}\left(\kappa_{v}\right)$.

Recall that the completion of $K$ with respect to a valuation $v$ is denoted by $K^{v}$.

Corollary 4.4. For $v \in \Omega(K)$ we have $p(K) \geq p\left(K^{v}\right)=p^{\prime}\left(\kappa_{v}\right)$.

Proof. Since $v$ extends to a $\mathbb{Z}$-valuation on $K^{v}$ with the same residue field $\kappa_{v}$, we obtain using both statements in Proposition 4.3 that $p(K) \geq p^{\prime}\left(\kappa_{v}\right)=p\left(K^{v}\right)$.

Corollary 4.5. We have $p^{\prime}(K(t))=p(K(t)) \geq p^{\prime}(K((t)))=p(K((t)))=p^{\prime}(K)$.

Proof. We have $p(K(t)) \geq p(K((t)))$ by Corollary 4.4 and $p^{\prime}(K((t)))=p(K((t)))=$ $p^{\prime}(K)$ by Proposition 4.3. If $K$ is real, then $K(t)$ is real, thus $p^{\prime}(K(t))=p(K(t))$ by the definition. If $K$ is nonreal, then $p(K(t))=s(K)+1=s(K(t))+1=p^{\prime}(K(t))$.

Corollary 4.6. Let $F / K$ be an algebrorational function field. Then $p^{\prime}(F)=p(F)$.

Proof. Replacing $K$ by its relative algebraic closure in $F$, we have $F=K(t)$ for some $t \in F$ transcendental over $K$. We conclude using Corollary 4.5.

This does not generalize to arbitrary algebraic function fields:

Example 4.7. Consider the function field $F$ of the curve $Y^{2}=-\left(X^{2}+1\right)\left(X^{2}+1+t\right)$ over $\mathbb{R}((t))$. By [Becher and Van Geel 2009, Example 5.13] we have $p(F)=s(F)=$ 2 , and therefore $p^{\prime}(F)=3>p(F)$. In particular $-1 \notin F^{\times 2}$ whereas -1 is a square in $F^{v}$ for any $v \in \Omega(F)$ by Corollary 4.4.

We apply Proposition 4.3 to give a short argument for a well-known fact: 
Corollary 4.8. Assume that $K$ is a finitely generated nonalgebraic extension of a subfield. Then $p(K) \geq 2$.

Proof. It follows from the hypotheses that there exists $v \in \Omega(K)$ such that $\kappa_{v}$ is nonreal. From Proposition 4.3 we obtain that $p(K) \geq p^{\prime}\left(\kappa_{v}\right)=s\left(\kappa_{v}\right)+1 \geq 2$.

Remark 4.9. If $K=k(t)$ for a subfield $k$ and $t \in K$ transcendental over $k$, then $1+t^{2} \notin K^{\times 2}$ and thus $p(K) \geq 2$. An alternative proof of Corollary 4.8 is therefore obtained by reduction to the case of a rational function field via the Diller-Dress Theorem [Lam 2005, Chapter VIII, Theorem 5.7], which says that if $p(K) \geq 2$ then $p(L) \geq 2$ for every finite field extension $L / K$.

For $S \subseteq \Omega(K)$ we define a homomorphism

$$
\Phi_{S}: K^{\times} \rightarrow \mathbb{Z}^{S}, \quad x \mapsto(v(x))_{v \in S} .
$$

If $S \subseteq \Omega(K)$ is a finite subset, then it follows from the Approximation Theorem (see [Engler and Prestel 2005, Theorem 2.4.1] or [Liu 2002, Chapter 9, Lemma 1.9]) that $\Phi_{S}$ is surjective.

The following statement extends Proposition 4.2 from a single $\mathbb{Z}$-valuation to finitely many $\mathbb{Z}$-valuations on $K$.

Proposition 4.10. Let $S$ be a finite subset of $\Omega(K)$ and $n \in \mathbb{N}$. Then

$$
\Phi_{S}\left(D_{K}(n)\right)=\left\{\left(e_{v}\right)_{v \in S} \in \mathbb{Z}^{S} \mid e_{v} \in 2 \mathbb{Z} \text { for } v \in S \text { with } s\left(\kappa_{v}\right) \geq n\right\} .
$$

Proof. For $v \in \Omega(K)$ with $s\left(\kappa_{v}\right) \geq n$ we have $v\left(D_{K}(n)\right) \subseteq 2 \mathbb{Z}$ by Lemma 4.1. This shows that

$$
\Phi_{S}\left(D_{K}(n)\right) \subseteq\left\{\left(e_{v}\right)_{v \in S} \in \mathbb{Z}^{S} \mid e_{v} \in 2 \mathbb{Z} \text { for } v \in S \text { with } s\left(\kappa_{v}\right) \geq n\right\} .
$$

It remains to show the other inclusion. Consider a tuple $\left(e_{v}\right)_{v \in S} \in \mathbb{Z}^{S}$ such that $e_{v} \in 2 \mathbb{Z}$ for all $v \in S$ with $s\left(\kappa_{v}\right) \geq n$. The aim is to find an element $x \in D_{K}(n)$ with $\Phi_{S}(x)=\left(e_{v}\right)_{v \in S}$. We explain how to obtain such an element, using the Approximation Theorem (see above) several times.

For $v \in S$ with $e_{v} \notin 2 \mathbb{Z}$, as $s\left(\kappa_{v}\right)<n$ we may choose $x_{v, 2}, \ldots, x_{v, n} \in \mathcal{O}_{v}$ such that $v\left(1+x_{v, 2}^{2}+\cdots+x_{v, n}^{2}\right)>0$. For $v \in S$ with $e_{v} \in 2 \mathbb{Z}$ we set $x_{v, 2}=\cdots=x_{v, n}=0$. For $i=2, \ldots, n$ we choose $x_{i} \in K^{\times}$such that $v\left(x_{i}-x_{v, i}\right)>0$ for all $v \in S$. We set $y=x_{2}^{2}+\cdots+x_{n}^{2}$. For $v \in S$ we have $v(1+y)=0$ if $e_{v} \in 2 \mathbb{Z}$ and $v(1+y)>0$ otherwise. We choose $t \in K^{\times}$such that, for all $v \in S$, we have $v(t)=1$ if $v(1+y)>1$, and $v(t)>1$ otherwise. Note that $(1+t)^{2}+y \in D_{K}(n)$. For any $v \in S$ the value $v\left((1+t)^{2}+y\right)$ is either 0 or 1 and such that $v\left((1+t)^{2}+y\right) \equiv e_{v} \bmod 2 \mathbb{Z}$. Now choose $z \in K^{\times}$such that $2 v(z)=e_{v}-v\left((1+t)^{2}+y\right)$ for all $v \in S$ and set $x=z^{2}\left((1+t)^{2}+y\right)$. Then $x \in D_{K}(n)$ and $\Phi_{S}(x)=(v(x))_{v \in S}=\left(e_{v}\right)_{v \in S}$. 
Corollary 4.11. Let $n \in \mathbb{N}$ and $S$ a finite subset of $\Omega(K)$ such that $s\left(\kappa_{v}\right)=2^{n}$ for all $v \in S$. Then $\Phi_{S}$ induces a surjective homomorphism

$$
D_{K}\left(2^{n+1}\right) / D_{K}\left(2^{n}\right) \rightarrow(\mathbb{Z} / 2 \mathbb{Z})^{S} .
$$

In particular, $\left|D_{K}\left(2^{n+1}\right) / D_{K}\left(2^{n}\right)\right| \geq 2^{|S|}$.

Proof. By the hypotheses on $S$ and by Proposition 4.10, we have $\Phi_{S}\left(D_{K}\left(2^{n+1}\right)\right)=$ $\mathbb{Z}^{S}$ and $\Phi_{S}\left(D_{K}\left(2^{n}\right)\right)=(2 \mathbb{Z})^{S}$. From this the statement follows.

Theorem 4.12. Let $K$ be a real field. For $n \in \mathbb{N}$ the following are equivalent:

(i) $p(K(X)) \leq 2^{n}$.

(ii) $p(L)<2^{n}$ for all finite real extensions $L / K$.

(iii) $s(L) \leq 2^{n-1}$ for all finite nonreal extensions $L / K$.

(iv) $p^{\prime}(L)<2^{n}$ for all finite extensions $L / K$ with $-1 \notin L^{\times 2}$.

Proof. See [Becher and Van Geel 2009, Theorem 3.3] for the equivalence of (i)-(iii); the equivalence of these conditions with (iv) is obvious.

Corollary 4.13. Let $n \in \mathbb{N}$ be such that $p(K(X)) \leq 2^{n}$. Then $p(L(X)) \leq 2^{n}$ for any finite field extension $L / K$.

Proof. If $K$ is nonreal, then $p(L(X))=s(L)+1 \leq s(K)+1=p(K(X)) \leq 2^{n}$. If $K$ is real and $L$ is nonreal, then $s(L) \leq 2^{n-1}$ by Theorem 4.12 and thus $p(L(X)) \leq 2^{n}$. If $L$ is real, then since any finite real extension of $L$ is a finite real extension of $K$, the equivalence of (i) and (ii) in Theorem 4.12 allows us to conclude that $p(L(X)) \leq 2^{n}$.

Theorem 4.14. Let $K$ be endowed with a $\mathbb{Z}$-valuation with residue field $k$. Then $p(K(X)) \geq p(k(X))$. Moreover, if the valuation is henselian and $n \in \mathbb{N}$ is such that $p(k(X)) \leq 2^{n}$, then $p(K(X)) \leq 2^{n}$.

Proof. Using Proposition 3.3 the given $\mathbb{Z}$-valuation on $K$ extends to a $\mathbb{Z}$-valuation on $K(X)$ with residue field $k(X)$. Hence, $p(K(X)) \geq p^{\prime}(k(X))=p(k(X))$ by Proposition 4.3 and Corollary 4.5.

Assume now that the $\mathbb{Z}$-valuation on $K$ is henselian. If $K$ is nonreal, then $p(K(X))=s(K)+1=s(k)+1=p(k(X))$. Assume that $K$ is real. Then $k$ and $k(X)$ are real. Let $n \in \mathbb{N}$ be such that $p(k(X)) \leq 2^{n}$. By Theorem 4.12, to prove that $p(K(X)) \leq 2^{n}$ it suffices to show that $p^{\prime}(L)<2^{n}$ for all finite extensions $L / K$ with $-1 \notin L^{\times 2}$. Consider such an extension $L / K$. Then $L$ is endowed with a henselian $\mathbb{Z}$-valuation whose residue field $\ell$ is a finite extension of $k$. Then $-1 \notin \ell^{\times 2}$ and thus $p^{\prime}(L)=p^{\prime}(\ell)<2^{n}$ by Proposition 4.3 and Theorem 4.12.

The last two statements motivate us to formulate the following two conjectures.

Conjecture 4.15. For any finite field extension $L / K$, one has $p(L(X)) \leq p(K(X))$. 
Conjecture 4.16. If $K$ is complete with respect to a nondyadic $\mathbb{Z}$-valuation with residue field $k$, then $p(K(X))=p(k(X))$.

Note that both conjectures hold trivially if $K$ is a nonreal field. In the case where $K$ is real, Conjecture 4.16 was raised originally by C. Scheiderer [2001, Remark 5.18.2] as a question. We shall prove in Corollary 6.9 that the two conjectures are equivalent.

\section{The $u$-invariant for algebraic function fields}

We refer to [Lam 2005] for basic facts and terminology from the theory of quadratic forms over fields of characteristic different from two. The $u$-invariant of $K$ was defined in [Elman and Lam 1973] as

$u(K)=\sup \{\operatorname{dim} \varphi \mid \varphi$ anisotropic torsion form over $K\} \in \mathbb{N} \cup\{\infty\}$,

where a torsion form is a regular quadratic form that corresponds to a torsion element in the Witt ring.

Proposition 5.1. Let $v \in \Omega(K)$. Let $\psi$ be a torsion form over $\kappa_{v}$. There exist $n \in \mathbb{N}$, $a_{1}, \ldots, a_{n} \in \mathbb{O}_{v}^{\times}$, and $t \in K^{\times}$with $v(t)=1$ such that $\langle 1,-t\rangle \otimes\left\langle a_{1}, \ldots, a_{n}\right\rangle$ is a torsion form over $K$ and such that $\psi$ is Witt equivalent to $\left\langle\bar{a}_{1}, \ldots, \bar{a}_{n}\right\rangle$.

Proof. Assume first that $v$ is nonreal. Then by Proposition 4.2 there exists $t \in \sum K^{2}$ with $v(t)=1$. For $n=\operatorname{dim} \psi$ and $a_{1}, \ldots, a_{n} \in \mathbb{O}_{v}^{\times}$such that $\psi$ is isometric to $\left\langle\bar{a}_{1}, \ldots, \bar{a}_{n}\right\rangle$, we obtain that $\langle 1,-t\rangle \otimes\left\langle a_{1}, \ldots, a_{n}\right\rangle$ is a torsion form over $K$.

Assume now that $v$ is real. Then $\psi$ is Witt equivalent to a sum of binary torsion forms over $\kappa_{v}$ (see [Pfister 1966, Satz 22]). Every binary torsion form over $\kappa_{v}$ is of the shape $\left\langle\bar{a}_{1}, \bar{a}_{2}\right\rangle$ with $a_{1}, a_{2} \in \mathbb{O}_{v}^{\times}$such that $-a_{1} a_{2} \in \sum K^{2}$. Hence, there exist $r \in \mathbb{N}$ and $a_{1}, \ldots, a_{2 r} \in \mathbb{O}_{v}^{\times}$such that $\psi$ is Witt equivalent to $\left\langle\bar{a}_{1}, \ldots, \bar{a}_{2 r}\right\rangle$ and $-a_{2 i-1} a_{2 i} \in \sum K^{2}$ for $i=1, \ldots, r$. Then $\left\langle a_{1}, \ldots, a_{2 r}\right\rangle$ is torsion form over $K$. We choose any $t \in K^{\times}$with $v(t)=1$. Then also $\langle 1,-t\rangle \otimes\left\langle a_{1}, \ldots, a_{2 r}\right\rangle$ is a torsion form over $K$.

The following statement was independently obtained in [Scheiderer 2009, Proposition 5] using different arguments, based on the theory of spaces of orderings.

Proposition 5.2. For $v \in \Omega(K)$ we have $u(K) \geq u\left(K^{v}\right)=2 u\left(\kappa_{v}\right)$.

Proof. Let $v \in \Omega(K)$. Let $\psi$ be an anisotropic torsion form over $\kappa_{v}$. Using Proposition 5.1 we choose $n \in \mathbb{N}, a_{1}, \ldots, a_{n} \in \mathcal{O}_{v}^{\times}$, and $t \in K^{\times}$with $v(t)=1$ such that $\psi$ is Witt equivalent to $\left\langle\bar{a}_{1}, \ldots, \bar{a}_{n}\right\rangle$ and such that $\langle 1,-t\rangle \otimes\left\langle a_{1}, \ldots, a_{n}\right\rangle$ is a torsion form over $K$. Let $\varphi$ denote its anisotropic part. Then $\varphi$ is a torsion form and isometric to $\left\langle b_{1}, \ldots, b_{s}\right\rangle \perp-t\left\langle c_{1}, \ldots, c_{r}\right\rangle$ for certain $r, s \in \mathbb{N}$ and $c_{1}, \ldots, c_{r}, b_{1}, \ldots, b_{s} \in \mathbb{O}_{v}^{\times}$. Applying residue homomorphisms (see [Lam 2005, Chapter VI, §1]), it follows that the forms $\left\langle\bar{b}_{1}, \ldots, \bar{b}_{s}\right\rangle$ and $\left\langle\bar{c}_{1}, \ldots, \bar{c}_{r}\right\rangle$ over $\kappa_{v}$ are 
Witt equivalent to $\psi$. As $\psi$ is anisotropic we conclude that $\operatorname{dim} \varphi=r+s \geq 2 \operatorname{dim} \psi$. This shows that $u(K) \geq 2 u\left(\kappa_{v}\right)$. Using Springer's Theorem for complete discretely valued fields (see [Lam 2005, Chapter VI, §1]), we further obtain that $u\left(K^{v}\right)=2 u\left(\kappa_{v}\right)$.

A generalization of Proposition 5.2 for arbitrary nondyadic valuations is given in [Becher and Leep 2013, Theorem 5.2].

Corollary 5.3. Let $k$ be the residue field of a nondyadic $\mathbb{Z}$-valuation on $K$. For every algebraic function field $F / K$ there exists an algebraic function field $E / k$ such that $u(F) \geq 2 u(E)$.

Proof. Let $T$ denote the discrete valuation ring with field of fractions $K$ and residue field $k$. Let $F / K$ be an algebraic function field. Choose $x \in F$ transcendental over $K$. Consider the valuation ring $T^{\prime}$ in $K(x)$ described in Proposition 3.3. Note that $T^{\prime}$ is a discrete valuation ring. Since $F / K(x)$ is a finite extension, there exists a $\mathbb{Z}$-valuation $v$ on $F$ dominating $T^{\prime}$. The residue field $E$ of $v$ is a finite extension of $k(\bar{x})$, hence an algebraic function field over $k$. By Proposition 5.2 we obtain that $u(F) \geq 2 u(E)$.

We define

$$
\hat{u}(K)=\frac{1}{2} \sup \{u(F) \mid F / K \text { algebraic function field }\} .
$$

For nonreal fields $\hat{u}$ coincides with the strong $u$-invariant defined in [Harbater et al. 2009, Definition 1.2], by the following result.

Corollary 5.4. For any algebraic extension $L / K$ we have

$$
u(L) \leq \frac{1}{2} u(K(X)) \leq \hat{u}(K) .
$$

Proof. If $L$ is a field of odd characteristic $p$, then the Frobenius homomorphism given by $x \longmapsto x^{p}$ shows that any quadratic form over $L$ is obtained by scalar extension from a quadratic form defined over $L^{p}$. Therefore every torsion form defined over an algebraic extension of $K$ comes from a torsion form defined over a finite separable extension of $K$. Since any finite separable extension of $K$ is the residue field of a $\mathbb{Z}$-valuation $v$ on $K(X)$, the first inequality now follows from Proposition 5.2. The second inequality is obvious.

\section{Function fields over complete discretely valued fields}

In this section we assume that $K$ is the field of fractions of a complete discrete valuation ring $T$ with residue field $k$ of characteristic different from 2 . We want to apply the following reformulation of the local-global principle in [Colliot-Thélène et al. 2012, Theorem 3.1] to the study of the $u$-invariant and the Pythagoras number of algebraic function fields over $K$. 
Theorem 6.1 (Colliot-Thélène, Parimala, Suresh). Let $F$ be an algebraic function field over $K$. A regular quadratic form over $F$ of dimension at least 3 is isotropic if and only if it is isotropic over $F^{v}$ for every $v \in \Omega(F)$.

Proof. This slightly more general version of the result cited follows from [Harbater et al. 2013, Proposition 9.10].

We will apply Theorem 6.1 to obtain upper bounds for the two mentioned field invariants. We have to distinguish two types of $\mathbb{Z}$-valuations on algebraic function fields over $K$.

Proposition 6.2. Let $F / K$ be an algebraic function field and $v \in \Omega(F)$. Then either $v$ is trivial on $K$ or it dominates $T$.

Proof. This follows from Proposition 2.2.

The lower bounds that we will obtain are based on more elementary arguments:

Lemma 6.3. Let $F / K$ be an algebraic function field and $v$ a ZZ-valuation on $F$ that is trivial on $K$. Then $p\left(F^{v}\right)=p^{\prime}\left(\kappa_{v}\right) \leq p(k(X))$ and $u\left(F^{v}\right)=2 u\left(\kappa_{v}\right) \leq u(k(X))$.

Proof. By Corollary 4.4 and Proposition 5.2 we have $p\left(F^{v}\right)=p^{\prime}\left(\kappa_{v}\right)$ and $u\left(F^{v}\right)=$ $2 u\left(\kappa_{v}\right)$. As $\kappa_{v}$ is a finite extension of $K$ and $T$ is a complete discrete valuation ring of $K$, there is a unique $\mathbb{Z}$-valuation $w$ on $\kappa_{v}$ with $O_{w} \cap K=T$. Then $\kappa_{w}$ is a finite extension of $k$, and $\kappa_{v}$ is complete with respect to $w$, in particular henselian. By Corollary 5.4 and Proposition 4.3 we obtain that $p^{\prime}\left(\kappa_{v}\right)=p^{\prime}\left(\kappa_{w}\right)$ and $u\left(\kappa_{v}\right)=2 u\left(\kappa_{w}\right)$. We choose $\alpha \in \kappa_{w}$ such that $\kappa_{w} / k(\alpha)$ is purely inseparable. Since $k$ is of characteristic different from 2, it follows that every element of $\kappa_{w}$ is a product of a square and an element from $k(\alpha)$. This yields that $p^{\prime}\left(\kappa_{w}\right) \leq p^{\prime}(k(\alpha))$ and $u\left(\kappa_{w}\right) \leq u(k(\alpha))$. Since $k(\alpha)$ is the residue field of a $\mathbb{Z}$-valuation on $k(X)$, we obtain from Proposition 4.3 and Corollary 5.4 that $p^{\prime}(k(\alpha)) \leq p(k(X))$ and $2 u(k(\alpha)) \leq u(k(X))$.

We can now extend Theorem 4.10 of [Harbater et al. 2009] to the current setting, thus covering real function fields. C. Scheiderer [2009, Theorem 3] independently gave a more geometric proof.

Theorem 6.4. We have $\hat{u}(K)=2 \hat{u}(k)$.

Proof. For any algebraic function field $E / k$, by Proposition 3.4 there exists an algebraic function field $F / K$ and a $\mathbb{Z}$-valuation on $F$ with residue field $E$, and using Proposition 5.2 we obtain that $u(E) \leq \frac{1}{2} u(F) \leq \hat{u}(K)$. This yields that $2 \hat{u}(k) \leq \hat{u}(K)$.

To prove the converse inequality, we need to show for an arbitrary algebraic function field $F / K$ that $u(F) \leq 4 \hat{u}(k)$ holds. Fix $F / K$. By Theorem 6.1, any anisotropic form over $F$ remains anisotropic over $F^{v}$ for some $v \in \Omega(F)$. It thus suffices to show that $u\left(F^{v}\right) \leq 4 \hat{u}(k)$ for every $v \in \Omega(F)$. Fix $v \in \Omega(F)$. As 
$u\left(F^{v}\right)=2 u\left(\kappa_{v}\right)$, it suffices to show that $u\left(\kappa_{v}\right) \leq 2 \hat{u}(k)$. If $v$ is trivial on $K$, we obtain by Lemma 6.3 that $2 u\left(\kappa_{v}\right) \leq u(k(X)) \leq 2 \hat{u}(k)$. Assume that $v$ is nontrivial on $K$. Then $\mathrm{O}_{v} \cap K=T$ by Proposition 6.2. If $\kappa_{v} / k$ is an algebraic function field then $u\left(\kappa_{v}\right) \leq 2 \hat{u}(k)$ by the definition of $\hat{u}(k)$. Otherwise $\kappa_{v} / k$ is an algebraic extension and then $u\left(\kappa_{v}\right) \leq \hat{u}(k)$ by Corollary 5.4.

Corollary 6.5. Let $m \in \mathbb{N}$. If $u(E)=m$ for every algebraic function field $E / k$, then $u(F)=2 m$ for every algebraic function field $F / K$.

Proof. Let $F / K$ be an algebraic function field over $K$. Using Theorem 6.4 we obtain that $u(F) \leq 2 \hat{u}(K)=4 \hat{u}(k)$. By Corollary 5.3 there exists an algebraic function field $E / k$ with $u(F) \geq 2 u(E)$. If we assume that $u(E)=m$ holds for every algebraic function field $E / k$, we obtain that $2 \hat{u}(k)=m$ and conclude that $u(F)=2 m$.

Theorem 6.6. We have that

$$
u(K(X))=2 \cdot \sup \{u(\ell(X)) \mid \ell / k \text { finite separable field extension }\} .
$$

Proof. Let $F=K(X)$. As $u(F) \geq 2$, it follows from Theorem 6.1 that

$$
u(F) \leq \sup \left\{u\left(F^{v}\right) \mid v \in \Omega(F)\right\} .
$$

Consider $v \in \Omega(F)$. If $v$ is trivial on $K$ then $u\left(F^{v}\right) \leq 2 u(k(X))$ by Lemma 6.3. If $v$ is nontrivial on $K$, then by Proposition 2.2 and Theorem $3.2 \kappa_{v} / k$ is either an algebraic extension or algebrorational. In any case we obtain that $u\left(\kappa_{v}\right) \leq u(\ell(X))$ and thus $u\left(F^{v}\right)=2 u\left(\kappa_{v}\right) \leq 2 u(\ell(X))$ for a finite extension $\ell / k$. Let $\ell^{\prime} / k$ be the separable subextension of $\ell / k$ such that $\ell / \ell^{\prime}$ is purely inseparable. Then $\ell(X) / \ell^{\prime}(X)$ is purely inseparable and of odd degree, so every element of $\ell(X)$ is a product of a square in $\ell(X)$ with an element of $\ell^{\prime}(X)$, whereby $u(\ell(X)) \leq u\left(\ell^{\prime}(X)\right)$. This together shows that

$$
u(F) \leq 2 \cdot \sup \{u(\ell(X)) \mid \ell / k \text { finite separable field extension }\} .
$$

On the other hand, given a finite separable field extension $\ell / k$, it follows from Theorem 3.5 that there exists a $\mathbb{Z}$-valuation on $F$ with residue field $\ell(X)$, which by Proposition 5.2 implies that $u(F) \geq 2 u(\ell(X))$. This shows the claimed equality.

We turn to the study of sums of squares and the Pythagoras number.

Theorem 6.7. Let $F / K$ be an algebraic function field. For any $m \geq 2$ we have that $D_{F}(m)=F^{\times} \cap\left(\bigcap_{v \in \Omega(F)} D_{F^{v}}(m)\right)$. Moreover, $p(F)=\sup \left\{p^{\prime}\left(\kappa_{v}\right) \mid v \in \Omega(F)\right\}$.

Proof. Applying Theorem 6.1 to the quadratic forms $m \times\langle 1\rangle \perp\langle-a\rangle$ for $a \in F^{\times}$ shows for any $m \geq 2$ the claimed equality of sets. Note that $\Omega(F)$ contains a 
nonreal valuation $v$, and we have that $p\left(F^{v}\right)=s\left(\kappa_{v}\right)+1 \geq 2$. Since $p(F) \geq 2$ by Corollary 4.8 , we obtain that

$$
\begin{aligned}
p(F) & =\inf \left\{m \geq 2 \mid D_{F}(m)=D_{F}(m+1)\right\} \\
& \leq \inf \left\{m \geq 2 \mid D_{F^{v}}(m)=D_{F^{v}}(m+1) \text { for all } v \in \Omega(F)\right\} \\
& =\sup \left\{p\left(F^{v}\right) \mid v \in \Omega(F)\right\} .
\end{aligned}
$$

Moreover, by Proposition 4.3 we have $p\left(F^{v}\right)=p^{\prime}\left(\kappa_{v}\right)$ for every $v \in \Omega(F)$.

Theorem 6.8. Let $F / K$ be an algebraic function field. There exists an algebraic function field $E / k$ such that $p^{\prime}(E) \geq p^{\prime}(F)$. Moreover, if $F / K$ is algebrorational, then one may choose $E / k$ to be algebrorational.

Proof. If $p(F)=\infty$, then as $F$ is a finite extension of a rational function field, we conclude with [Pfister 1995, Chapter 7, Proposition 1.13] that $p(K(X))=\infty$ and then with Theorem 4.14 we obtain that $p(k(X))=\infty$, so that for $E=k(X)$ we have $p^{\prime}(E)=\infty=p^{\prime}(F)$.

We now suppose that $p(F)<\infty$. By Theorem 6.7 there exists $v \in \Omega(F)$ such that $p(F)=p^{\prime}\left(\kappa_{v}\right)$.

Assume first that $p^{\prime}(F) \neq p(F)$. Then $F$ is nonreal with $p(F)=s(F)$, and by Corollary $4.6 F$ is not algebrorational. It follows that $\kappa_{v}$ is nonreal with $s\left(\kappa_{v}\right)=$ $p^{\prime}\left(\kappa_{v}\right)-1=p(F)-1=s(F)-1$, and as $s\left(\kappa_{v}\right)$ and $s(F)$ are both powers of two, we conclude that $s(F)=2$. Then $s(k) \geq 2$ and for $E=k(X)\left(\sqrt{-\left(1+X^{2}\right)}\right)$ we have that $s(E)=2$ and thus $p^{\prime}(E)=3=p^{\prime}(F)$.

Suppose now that $p^{\prime}(F)=p(F)=p^{\prime}\left(\kappa_{v}\right)$. If $\left.v\right|_{K}$ is trivial, then we have $p(k(X)) \geq p^{\prime}\left(\kappa_{v}\right)=p(F)$ by Lemma 6.3 and further $s(k(X))=s(k)=s(K) \geq s(F)$, so we may choose $E=k(X)$ to have $p^{\prime}(E) \geq p^{\prime}(F)$. Suppose that $\left.v\right|_{K}$ is nontrivial. By Proposition 6.2 then $v$ dominates $T$, and the residue extension $\kappa_{v} / k$ is either algebraic or it is an algebraic function field. If $\kappa_{v} / k$ is an algebraic function field, we may choose $E=\kappa_{v}$ and have that $p^{\prime}(E) \geq p^{\prime}(F)$. Moreover, by Theorem 3.2, if $F / K$ is algebrorational, then so is $E / k$. If $\kappa_{v} / k$ is algebraic, then as $p^{\prime}\left(\kappa_{v}\right)=$ $p^{\prime}(F)<\infty$ there exists a finite extension $\ell / k$ contained in $\kappa_{v} / k$ with $p^{\prime}(\ell) \geq p^{\prime}\left(\kappa_{v}\right)$, and we may thus choose $E=\ell(X)$ to have $p^{\prime}(E) \geq p^{\prime}(\ell) \geq p^{\prime}\left(\kappa_{v}\right)=p^{\prime}(F)$.

Corollary 6.9. We have $p(K(X))=\sup \{p(\ell(X)) \mid \ell / k$ finite field extension $\}$.

Proof. The statement is trivial if $k$ is nonreal. Assume that $k$ is real. Given an arbitrary finite extension $\ell / k$, by Theorem 3.5 there is a $\mathbb{Z}$-valuation on $K(X)$ with residue field $\ell(X)$, whereby Proposition 4.3 yields that $p^{\prime}(\ell(X)) \leq p^{\prime}(K(X))$. On the other hand, by Theorem 6.8, there exists a finite extension $\ell / k$ with $p^{\prime}(K(X)) \leq$ $p^{\prime}(\ell(X))$. Since $p^{\prime}(K(X))=p(K(X))$ and $p^{\prime}(k(X))=p(k(X))$ the statement follows.

Note that Corollary 6.9 shows the equivalence of Conjectures 4.15 and 4.16. 
Theorem 6.10. Let $n \in \mathbb{N}$. Assume that $p(k(X)) \leq 2^{n}$ and that $\sum E^{2} / D_{E}\left(2^{n}\right)$ is finite for every algebraic function field $E / k$. Then $p(K(X)) \leq 2^{n}$ and $\sum F^{2} / D_{F}\left(2^{n}\right)$ is finite for every algebraic function field $F / K$.

Proof. By Theorem 4.14 we have $p(K(X)) \leq 2^{n}$. Consider an algebraic function field $F / K$. By Theorem 6.7 the natural homomorphism

$$
\sum F^{2} / D_{F}\left(2^{n}\right) \longrightarrow \prod_{v \in \Omega(F)} \sum\left(F^{v}\right)^{2} / D_{F}\left(2^{n}\right)
$$

is injective. To prove that $\sum F^{2} / D_{F}\left(2^{n}\right)$ is finite, it thus suffices to show that the set

$$
S=\left\{v \in \Omega(F) \mid p\left(F^{v}\right)>2^{n}\right\}
$$

is finite and that $\sum\left(F^{v}\right)^{2} / D_{F^{v}}\left(2^{n}\right)$ is finite for each $v \in S$. Consider $v \in \Omega(F)$. If $v$ is trivial on $K$, then $p\left(F^{v}\right) \leq p(k(X)) \leq 2^{n}$ by Lemma 6.3. Otherwise $\mathcal{O}_{v} \cap K=T$ by Proposition 6.2 and $\kappa_{v}$ is an extension of $k$. If the extension $\kappa_{v} / k$ is algebraic, then $p\left(F^{v}\right)=p^{\prime}\left(\kappa_{v}\right) \leq p(k(X)) \leq 2^{n}$. If $\kappa_{v} / k$ is an algebraic function field which is algebrorational, then using Corollary 6.9 we obtain that $p\left(F^{v}\right)=p^{\prime}\left(\kappa_{v}\right) \leq p(K(X)) \leq 2^{n}$. This proves that, for any $v \in S$, we have $\mathrm{O}_{v} \cap K=T$ and $\kappa_{v} / k$ is an algebraic function field that is not algebrorational. The finiteness of $S$ thus follows from Corollary 3.9, and for any $v \in S$ we have $\left|\sum\left(F^{v}\right)^{2} / D_{F^{v}}\left(2^{n}\right)\right| \leq 2 \cdot\left|\sum\left(\kappa_{v}\right)^{2} / D_{\kappa_{v}}\left(2^{n}\right)\right|$, which is finite by the hypothesis.

Theorem 6.11. Assume that $n \in \mathbb{N}$ is such that $p(E) \leq 2^{n}$ for any algebraic function field $E / k$. Let $F / K$ be an algebraic function field. Then $p(F) \leq 2^{n}+1$ and the set $S=\left\{v \in \Omega(F) \mid s\left(\kappa_{v}\right)=2^{n}\right\}$ is finite with $\left|\sum F^{2} / D_{F}\left(2^{n}\right)\right|=2^{|S|}$. Moreover, $\Phi_{S}: F^{\times} \rightarrow \mathbb{Z}^{S}$ induces an isomorphism $\sum F^{2} / D_{F}\left(2^{n}\right) \rightarrow(\mathbb{Z} / 2 \mathbb{Z})^{S}$.

Proof. Consider $v \in \Omega(F)$. If $\left.v\right|_{K}$ is trivial, then $p^{\prime}\left(\kappa_{v}\right) \leq p(k(X)) \leq 2^{n}$ by Lemma 6.3 and the hypothesis. Suppose now that $\left.v\right|_{K}$ is nontrivial. By Proposition 6.2 then $O_{v} \cap K=T$ and the residue field extension $\kappa_{v} / k$ is either algebraic or it is an algebraic function field. If $\kappa_{v} / k$ is algebraic, then $p^{\prime}\left(\kappa_{v}\right) \leq 2^{n}$. Suppose that $\kappa_{v} / k$ is an algebraic function field. Then $p\left(\kappa_{v}\right) \leq 2^{n}$ by the hypothesis. Moreover, if $\kappa_{v} / k$ is algebrorational, then Corollary 4.6 yields that $p^{\prime}\left(\kappa_{v}\right)=p\left(\kappa_{v}\right) \leq 2^{n}$.

Hence, in any case we have that $p\left(\kappa_{v}\right) \leq 2^{n}$, and thus $p\left(F^{v}\right)=p^{\prime}\left(\kappa_{v}\right) \leq 2^{n}+1$ by Corollary 4.4. Furthermore, we conclude that $p\left(F^{v}\right)=2^{n}+1$ if and only if $v \in S$, and in this case the residue field extension $\kappa_{v} / k$ is an algebraic function field but not algebrorational.

By Theorem 6.7 we conclude that $p(F) \leq p^{\prime}(F) \leq 2^{n}+1$ and furthermore

$$
\sum F^{2}=\left(\bigcap_{v \in S} D_{F^{v}}\left(2^{n}+1\right)\right) \cap\left(\bigcap_{v \in S^{c}} D_{F^{v}}\left(2^{n}\right)\right) \cap F^{\times},
$$


where $S^{\mathrm{c}}=\Omega(F) \backslash S$. Moreover, using Corollary 3.9 we obtain that $S$ is finite. By Corollary 4.11 then $\Phi_{S}: F^{\times} \rightarrow \mathbb{Z}^{S}$ induces a surjective homomorphism $\sum F^{2} / D_{F}\left(2^{n}\right) \rightarrow(\mathbb{Z} / 2 \mathbb{Z})^{S}$. It remains to show that this homomorphism is also injective. In view of Theorem 6.7 and the above equality for $\sum F^{2}$, it suffices to verify that $\Phi_{S}^{-1}\left((2 \mathbb{Z})^{S}\right) \subseteq \bigcap_{v \in S} D_{F^{v}}\left(2^{n}\right)$. Consider $x \in \sum F^{2}$ and $v \in S$ with $v(x) \in 2 \mathbb{Z}$. Then $x=t^{2} y$ with $t \in F^{\times}$and $y \in \mathcal{O}_{v}^{\times} \cap\left(\sum F^{2}\right)$, whereby $y+\mathfrak{m}_{v} \in \sum \kappa_{v}{ }^{2}$. Since $F^{v}$ is complete and $p\left(\kappa_{v}\right) \leq 2^{n}$, it follows that $x=t^{2} y \in D_{F^{v}}\left(2^{n}\right)$. This shows the claim.

Recall that the field $K$ is said to be hereditarily quadratically closed if $L^{\times}=L^{\times 2}$ for every finite field extension $L / K$. The following result applies in particular to the situation where $R$ is a real closed field.

Theorem 6.12. Let $n \in \mathbb{N}$ and $K=R\left(\left(t_{1}\right)\right)$... ((t $\left.\left.t_{n}\right)\right)$ for a field $R$ such that $R(\sqrt{-1})$ is hereditarily quadratically closed. Let $F / K$ be an algebraic function field. Then $u(F)=2^{n+1}, 2 \leq p(F) \leq 3$, and the group $\sum F^{2} / D_{F}(2)$ is finite.

Proof. We prove this by induction on $n$. For $n=0$ we obtain from [Elman and Wadsworth 1987, Theorem] that $u(F)=2$, and we conclude by [Lam 2005, Chapter XI, Corollary 6.26] and Corollary 4.8 that $p(F)=2$, whereby $\sum F^{2}=$ $D_{F}(2)$ and $2 \leq p(F) \leq p^{\prime}(F) \leq 3$. Assume that $n>0$. Applying the induction hypothesis to all algebraic function fields over $k=R\left(\left(t_{1}\right)\right) \ldots\left(\left(t_{n-1}\right)\right)$, we obtain by Corollary 6.5 that $u(F)=2^{n+1}$, by Corollary 4.8 and Theorem 6.8 that $2 \leq p(F) \leq$ $p^{\prime}(F) \leq 3$, and by Theorem 6.10 that $\sum F^{2} / D_{F}(2)$ is finite.

For certain real function fields over $\mathbb{R}((t))$, it was asked in [Becher and Van Geel 2009, Question 5.15] whether their Pythagoras number is three or four. We can now answer this question:

Corollary 6.13. Let $h \in \mathbb{R}[X]$ be a nonconstant square-free polynomial with no roots in $\mathbb{R}$. Let $F$ be the function field of the curve $Y^{2}=(t X-1) h$ over $\mathbb{R}((t))$. Then $p(F)=3$.

Proof. We have $p(F) \geq 3$ by [Becher and Van Geel 2009, Theorem 5.3 and Corollary 4.2] and $p(F) \leq 3$ by Theorem 6.12 .

\section{Acknowledgments}

We wish to thank Jean-Louis Colliot-Thélène and Yong Hu for helpful discussions in the context of Proposition 3.7, and furthermore Arno Fehm and Claus Scheiderer for comments on the text. We further wish to acknowledge the scrutiny of the referee, whose comments helped us to improve the presentation. This work was supported by the Deutsche Forschungsgemeinschaft (project Quadratic Forms and Invariants, BE 2614/3), by the Swiss National Science Foundation (Grant 200020-124785/1), and by the Zukunftskolleg, Universität Konstanz. 


\section{References}

[Abhyankar 1956] S. Abhyankar, "On the valuations centered in a local domain", Amer. J. Math. 78 (1956), 321-348. MR 18,556b Zbl 0074.26301

[Becher and Leep 2013] K. J. Becher and D. B. Leep, "Real fields, valuations, and quadratic forms", Manuscripta Math. 141:3-4 (2013), 737-754. MR 3062606 Zbl 06179653

[Becher and Van Geel 2009] K. J. Becher and J. Van Geel, "Sums of squares in function fields of hyperelliptic curves”, Math. Z. 261:4 (2009), 829-844. MR 2009m:12001 Zbl 1253.11050

[Colliot-Thélène et al. 2012] J.-L. Colliot-Thélène, R. Parimala, and V. Suresh, "Patching and localglobal principles for homogeneous spaces over function fields of $p$-adic curves", Comment. Math. Helv. 87:4 (2012), 1011-1033. MR 2984579 Zbl 06104833

[Elman and Lam 1973] R. Elman and T. Y. Lam, "Quadratic forms and the $u$-invariant, I", Math. Z. 131 (1973), 283-304. MR 48 \#2072 Zbl 0244.10020

[Elman and Wadsworth 1987] R. Elman and A. R. Wadsworth, "Hereditarily quadratically closed fields", J. Algebra 111:2 (1987), 475-482. MR 89g:12004 Zbl 0669.12010

[Engler and Prestel 2005] A. J. Engler and A. Prestel, Valued fields, Springer, Berlin, 2005. MR 2007a: 12005 Zbl 1128.12009

[Harbater et al. 2009] D. Harbater, J. Hartmann, and D. Krashen, "Applications of patching to quadratic forms and central simple algebras”, Invent. Math. 178:2 (2009), 231-263. MR 2010j:11058 Zbl 1259.12003

[Harbater et al. 2013] D. Harbater, J. Hartmann, and D. Krashen, "Local-global principles for torsors over arithmetic curves", preprint, 2013. In versions 1 and 2 (dated 2011) the result cited appears as 9.9 rather than 9.10. arXiv $1108.3323 \mathrm{v} 4$

[Lam 2005] T. Y. Lam, Introduction to quadratic forms over fields, Graduate Studies in Mathematics 67, American Mathematical Society, Providence, RI, 2005. MR 2005h:11075 Zbl 1068.11023

[Liu 2002] Q. Liu, Algebraic geometry and arithmetic curves, Oxford Graduate Texts in Mathematics 6, Oxford University Press, 2002. MR 2003g:14001 Zbl 0996.14005

[Matsumura 1986] H. Matsumura, Commutative ring theory, Cambridge Studies in Advanced Mathematics 8, Cambridge University Press, 1986. MR 88h:13001 Zbl 0603.13001

[Nagata 1967] M. Nagata, "A theorem on valuation rings and its applications", Nagoya Math. J. 29 (1967), 85-91. MR 34 \#7503 Zbl 0146.26302

[Ohm 1983] J. Ohm, "The ruled residue theorem for simple transcendental extensions of valued fields", Proc. Amer. Math. Soc. 89:1 (1983), 16-18. MR 85b:12010 Zbl 0523.12021

[Parimala and Suresh 2010] R. Parimala and V. Suresh, "The $u$-invariant of the function fields of p-adic curves", Ann. of Math. (2) 172:2 (2010), 1391-1405. MR 2011g:11074 Zbl 1208.11053

[Pfister 1966] A. Pfister, "Quadratische Formen in beliebigen Körpern”, Invent. Math. 1 (1966), 116-132. MR 34 \#169 Zbl 0142.27203

[Pfister 1995] A. Pfister, Quadratic forms with applications to algebraic geometry and topology, London Mathematical Society Lecture Note Series 217, Cambridge University Press, 1995. MR 97c:11046 Zbl 0847.11014

[Scheiderer 2001] C. Scheiderer, "On sums of squares in local rings", J. Reine Angew. Math. 540 (2001), 205-227. MR 2002j:13031 Zbl 0991.13014

[Scheiderer 2009] C. Scheiderer, "The $u$-invariant of one-dimensional function fields over real power series fields", Arch. Math. (Basel) 93:3 (2009), 245-251. MR 2011d:11090 Zbl 1241.11047 
Received June 22, 2012. Revised December 2, 2013.

KARIM JOHANNES BECHER

DEPARTEMENT WISKUNDE EN INFORMATICA

UNIVERSITEIT ANTWERPEN

MIDDELHEIMLAAN 1

2020 ANTWERP

BELGIUM

and

ZUKUNFTSKOLLEG

UNIVERSITÄT KONSTANZ

Box 216

78457 KONSTANZ

GERMANY

becher@maths.ucd.ie

DAVID GRIMM

École Polytechnique Fédérale de LAUSANNE

EPFL-SB-MATHGEOM-CSAG

STATION 8

CH-1015 LAUSANNE

SWITZERLAND

david.grimm@epfl.ch

JAN VAN GEEL

UNIVERSITEIT GENT

VAKGROEP WISKUNDE

KRIJGSLAAN 281

9000 GHENT

BELGIUM

jvg@cage.ugent.be 


\title{
PACIFIC JOURNAL OF MATHEMATICS
}

\author{
msp.org/pjm
}

Founded in 1951 by E. F. Beckenbach (1906-1982) and F. Wolf (1904-1989)

\section{EDITORS}

V. S. Varadarajan (Managing Editor)

Department of Mathematics

University of California

Los Angeles, CA 90095-1555

pacific@math.ucla.edu

Paul Balmer

Department of Mathematics

University of California

Los Angeles, CA 90095-1555

balmer@math.ucla.edu

Daryl Cooper

Department of Mathematics

University of California

Santa Barbara, CA 93106-3080 cooper@math.ucsb.edu

Jiang-Hua $\mathrm{Lu}$

Department of Mathematics

Pokfulam Rd., Hong Kong jhlu@maths.hku.hk
The University of Hong Kong

Don Blasius

Department of Mathematics University of California

Los Angeles, CA 90095-1555

blasius@math.ucla.edu

Robert Finn

Department of Mathematics Stanford University

Stanford, CA 94305-2125

finn@math.stanford.edu

Sorin Popa

Department of Mathematics

University of California

Los Angeles, CA 90095-1555 popa@math.ucla.edu

Paul Yang

Department of Mathematics Princeton University

Princeton NJ 08544-1000

yang@math.princeton.edu

\section{PRODUCTION}

Silvio Levy, Scientific Editor, production@msp.org

\section{SUPPORTING INSTITUTIONS}

ACADEMIA SINICA, TAIPEI

CALIFORNIA INST. OF TECHNOLOGY

INST. DE MATEMÁTICA PURA E APLICADA

KEIO UNIVERSITY

MATH. SCIENCES RESEARCH INSTITUTE

NEW MEXICO STATE UNIV.

OREGON STATE UNIV.

\author{
STANFORD UNIVERSITY \\ UNIV. OF BRITISH COLUMBIA \\ UNIV. OF CALIFORNIA, BERKELEY \\ UNIV. OF CALIFORNIA, DAVIS \\ UNIV. OF CALIFORNIA, LOS ANGELES \\ UNIV. OF CALIFORNIA, RIVERSIDE \\ UNIV. OF CALIFORNIA, SAN DIEGO \\ UNIV. OF CALIF., SANTA BARBARA
}

\author{
Vyjayanthi Chari \\ Department of Mathematics \\ University of California \\ Riverside, CA 92521-0135 \\ chari@math.ucr.edu \\ Kefeng Liu \\ Department of Mathematics \\ University of California \\ Los Angeles, CA 90095-1555 \\ liu@math.ucla.edu \\ Jie Qing \\ Department of Mathematics \\ University of California \\ Santa Cruz, CA 95064 \\ qing@cats.ucsc.edu
}

These supporting institutions contribute to the cost of publication of this Journal, but they are not owners or publishers and have no responsibility for its contents or policies.

See inside back cover or msp.org/pjm for submission instructions.

The subscription price for 2014 is US \$410/year for the electronic version, and \$535/year for print and electronic.

Subscriptions, requests for back issues and changes of subscribers address should be sent to Pacific Journal of Mathematics, P.O. Box 4163, Berkeley, CA 94704-0163, U.S.A. The Pacific Journal of Mathematics is indexed by Mathematical Reviews, Zentralblatt MATH, PASCAL CNRS Index, Referativnyi Zhurnal, Current Mathematical Publications and Web of Knowledge (Science Citation Index).

The Pacific Journal of Mathematics (ISSN 0030-8730) at the University of California, c/o Department of Mathematics, 798 Evans Hall \#3840, Berkeley, CA 94720-3840, is published twelve times a year. Periodical rate postage paid at Berkeley, CA 94704, and additional mailing offices. POSTMASTER: send address changes to Pacific Journal of Mathematics, P.O. Box 4163, Berkeley, CA 94704-0163.

PJM peer review and production are managed by EditFLOW ${ }^{\circledR}$ from Mathematical Sciences Publishers.

\section{PUBLISHED BY}

mathematical sciences publishers

nonprofit scientific publishing

http://msp.org/

(C) 2014 Mathematical Sciences Publishers 


\section{PACIFIC JOURNAL OF MATHEMATICS}

Volume $267 \quad$ No. $2 \quad$ February 2014

Sums of squares in algebraic function fields over a complete discretely valued field

KARIM JOHANNES BECHER, DAVID GRIMM and JAN VAN GEEL

On the equivalence problem for toric contact structures on $\mathbf{S}^{\mathbf{3}}$-bundles over $S^{2}$

\section{Charles P. Boyer and Justin PATi}

An almost-Schur type lemma for symmetric $(2,0)$ tensors and applications

Xu CHENG

Algebraic invariants, mutation, and commensurability of link complements

ERIC CHESEBRO and JASON DEBLOIS

Taut foliations and the action of the fundamental group on leaf spaces and universal circles

YOSUKE KANO

A new monotone quantity along the inverse mean curvature flow in $\mathbb{R}^{n}$

KwoK-Kun Kwong and Pengzi Miao

Nonfibered L-space knots

TYE LIDMAN and LIAM WATSON

Families and Springer's correspondence

GEORGE LUSZTIG

Reflexive operator algebras on Banach spaces

Florence Merlevède, Costel Peligrad and Magda Peligrad Harer stability and orbifold cohomology

NiCOLA PAGANI

Spectra of product graphs and permanents of matrices over finite rings

LE ANH VINH

The concavity of the Gaussian curvature of the convex level sets of minimal surfaces with respect to the height

PEI-HE WANG 